\title{
A RADIO-SELECTED BLACK HOLE X-RAY BINARY CANDIDATE IN THE MILKY WAY GLOBULAR CLUSTER M62
}

\author{
Laura Chomiuk $^{1,2}$, Jay Strader ${ }^{2}$, Thomas J. Maccarone ${ }^{3}$, James C. A. Miller-Jones ${ }^{4}$, \\ Craig Heinke ${ }^{5}$, Eva Noyola ${ }^{6}$, Anil C. Seth $^{7}$, and Scott Ransom ${ }^{1}$ \\ ${ }^{1}$ National Radio Astronomy Observatory, 520 Edgemont Rd, Charlottesville, VA, USA; chomiuk@pa.msu.edu \\ ${ }^{2}$ Department of Physics and Astronomy, Michigan State University, East Lansing, MI 48824, USA \\ ${ }^{3}$ Department of Physics, Texas Tech University, Box 41051, Lubbock, TX 79409-1051, USA \\ ${ }^{4}$ International Centre for Radio Astronomy Research, Curtin University, GPO Box U1987, Perth, WA 6845, Australia \\ ${ }^{5}$ Department of Physics, University of Alberta, 4-183 CCIS, Edmonton, AB T6G 2E1, Canada \\ ${ }^{6}$ Instituto de Astronomia, Universidad Nacional Autonoma de Mexico (UNAM), A. P. 70-264, 04510, Mexico \\ ${ }^{7}$ Department of Physics and Astronomy, University of Utah, Salt Lake City, UT 84112, USA \\ Received 2013 June 10; accepted 2013 September 4; published 2013 October 17
}

\begin{abstract}
We report the discovery of a candidate stellar-mass black hole in the Milky Way globular cluster M62. We detected the black hole candidate, which we call M62-VLA1, in the core of the cluster using deep radio continuum imaging from the Karl G. Jansky Very Large Array. M62-VLA1 is a faint source with a flux density of $18.7 \pm 1.9 \mu \mathrm{Jy}$ at $6.2 \mathrm{GHz}$ and a flat radio spectrum $\left(\alpha=-0.24 \pm 0.42\right.$, for $\left.S_{v}=v^{\alpha}\right)$. M62 is the second Milky Way cluster with a candidate stellar-mass black hole; unlike the two candidate black holes previously found in the cluster M22, M62-VLA1 is associated with a Chandra X-ray source, supporting its identification as a black hole X-ray binary. Measurements of its radio and X-ray luminosity, while not simultaneous, place M62-VLA1 squarely on the well-established radio-X-ray correlation for stellar-mass black holes. In archival Hubble Space Telescope imaging, M62-VLA1 is coincident with a star near the lower red giant branch. This possible optical counterpart shows a blue excess, $\mathrm{H} \alpha$ emission, and optical variability. The radio, X-ray, and optical properties of M62-VLA1 are very similar to those for V404 Cyg, one of the best-studied quiescent stellar-mass black holes. We cannot yet rule out alternative scenarios for the radio source, such as a flaring neutron star or background galaxy; future observations are necessary to determine whether M62-VLA1 is indeed an accreting stellar-mass black hole.
\end{abstract}

Key words: black hole physics - globular clusters: individual (M62) - radio continuum: general - X-rays: general

Online-only material: color figures

\section{INTRODUCTION}

For a typical globular cluster (GC) with a present day mass of $\sim 10^{5}-10^{6} M_{\odot}$, hundreds of stellar-mass black holes (BHs) should be born during the first $\sim 10 \mathrm{Myr}$ after formation (Larson 1984; Kulkarni et al. 1993). These BHs have masses $\sim 5-20 M_{\odot}$ and mark the endpoints of stars with main-sequence masses $\gtrsim 25 M_{\odot}$ (Heger et al. 2003). The specific frequency of X-ray binaries in GCs is $\sim 100$ times larger than the field and provides strong evidence that mass-transferring binaries are dynamically formed with high efficiency in GCs (e.g., Kundu et al. 2002; Pooley et al. 2003). If BHs are present in GCs, a subset should be detectable as accreting binaries.

Yet, soon after the detection of luminous X-ray sources in Milky Way GCs, it became clear that accreting neutron stars, not $\mathrm{BHs}$, dominate luminous cluster X-ray binaries. The principal evidence is the detection of Type I X-ray bursts that are wellexplained by thermonuclear runaway events on the surfaces of neutron stars (Lewin \& Joss 1981). Of the 18 luminous Galactic GC X-ray sources published so far, all are thought to contain neutron stars, due to the presence of X-ray bursts (in 15; see, e.g., Verbunt \& Lewin 2006; Altamirano et al. 2012), coherent pulsations (in 4; e.g., Altamirano et al. 2010), optical spectroscopy (van Zyl et al. 2004), or a low radio-to-X-ray flux ratio (Bozzo et al. 2011; see below for the rationale).

Theoretical expectations for the presence of $\mathrm{BHs}$ in GCs remain uncertain. After birth, assuming that BHs receive small natal kicks, they will rapidly mass segregate to the cluster center in a fraction of a relaxation time and form a subcluster that is dynamically segregated from the rest of the GC. Many BH-BH binaries will then be formed through three-body interactions, and these binaries halt the collapse of the subcluster through interactions with one another. This process will tend to harden the binaries and lead to interactions with large recoil velocities, ejecting many BHs from the GC. Once a sufficient number of BHs have been ejected, the subcluster will no longer be dynamically decoupled from the stars in the core of the cluster, and the efficiency of $\mathrm{BH}$ ejection will decrease.

The eventual fate of the remaining BHs is poorly understood. Many papers have suggested that, at present, GCs should have at most one $\mathrm{BH}$ (or $\mathrm{BH}-\mathrm{BH}$ binary) remaining and that many clusters should have none (Kulkarni et al. 1993; Sigurdsson \& Hernquist 1993; Portegies Zwart \& McMillan 2000; Kalogera et al. 2004). Some recent papers have argued that a few BHs may be retained (Mackey et al. 2008; Moody \& Sigurdsson 2009; Aarseth 2012), while two new theory papers suggest that in some circumstances many BHs could survive in GCs (Sippel \& Hurley 2013; Morscher et al. 2013). In sum, recent work supports the idea that BH ejection in GCs is less efficient than previously thought.

Over the past $\sim 6 \mathrm{yr}$, there have been solid BH candidates identified in GCs around other galaxies (e.g., Maccarone et al. 2007; Irwin et al. 2010). These sources are characterized as likely BH binaries because (1) they have X-ray luminosities far above the Eddington luminosity for a single neutron star, and (2) they vary significantly on short timescales, making it implausible that the luminosity originates from a superposition of several neutron star X-ray binaries. The most luminous of 

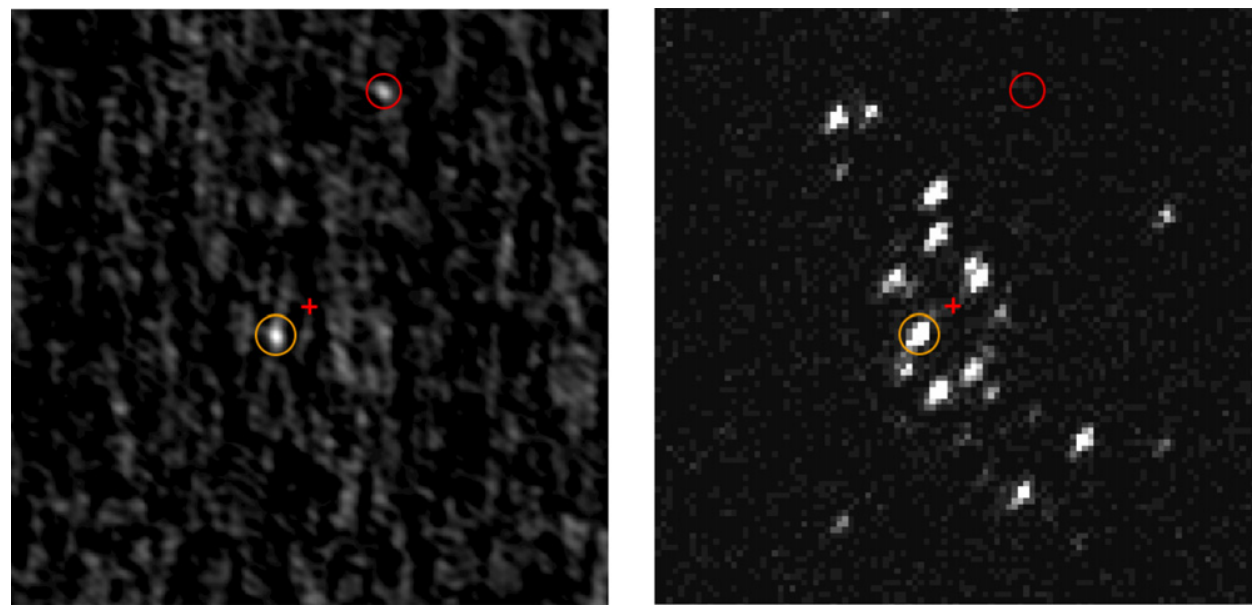

Figure 1. VLA 6.2 GHz radio (left) and Chandra X-ray (right) images of the central 50" (1.6 pc) of M62, showing the candidate BH M62-VLA1 (orange circle). A red cross marks the cluster photometric center (Lützgendorf et al. 2013). The other radio source in the central region of M62 is a known pulsar (red circle; Possenti et al. 2003). North is up and east is to the left.

(A color version of this figure is available in the online journal.)

these objects is associated with a GC in the massive Virgo elliptical NGC 4472, and has a peak $L_{X} \sim 4 \times 10^{39} \mathrm{erg} \mathrm{s}^{-1}$ (Maccarone et al. 2007). Optical spectroscopy of the associated GC shows broad (1500 $\mathrm{km} \mathrm{s}^{-1}$ ) [O III] emission but no Balmer lines, and the combined data is best explained by a model in which a stellar-mass $\mathrm{BH}$ is accreting at a super-Eddington rate from a CO white dwarf (Zepf et al. 2008; Peacock et al. 2012).

The few observed super-Eddington BHs in extragalactic GCs would then simply be those with the most extreme accretion rates. They likely represent only the very tip of the iceberg in terms of BH binaries in GCs. Many BHs with lower accretion rates are almost certain to exist among X-ray sources in GCs, but they are greatly outnumbered by neutron-star binaries and are difficult to distinguish from neutron stars using X-ray data alone.

In Strader et al. (2012a), we developed a new strategy for identifying quiescent $\mathrm{BH}$ binaries in Milky Way GCs, making use of both radio and X-ray data (see also Maccarone \& Knigge 2007). Stellar-mass BHs accreting at low rates have compact jets which emit radio continuum via partially selfabsorbed synchrotron emission (Blandford \& Königl 1979). Thus, they are much more luminous in the radio than neutron stars with comparable X-ray luminosity: $L_{R} / L_{X}$ is $\sim 2$ orders of magnitude higher for BHs than neutron stars (Migliari \& Fender 2006). Before the recent upgrade to the VLA, the radio emission from a quiescent $\mathrm{BH}$ like A0620-00 or V404 Cyg would not have been detectable at high significance at typical GC distances (Gallo et al. 2006). The upgraded VLA can now readily detect the expected flux densities (tens of $\mu \mathrm{Jy}$ ) in reasonable exposure times.

Using this technique, we discovered two candidate stellarmass BHs in the cluster core of M22 (Strader et al. 2012a). The sources have flat radio spectra and $6 \mathrm{GHz}$ flux densities of $55-60 \mu \mathrm{Jy}$. As these sources are not detected in shallow archival Chandra imaging, they cannot yet be placed directly on the $L_{X}-L_{R}$ relation; nevertheless, their overall properties are consistent with those expected from accreting $\mathrm{BH}$ binaries.

Here we report the discovery of a $\mathrm{BH}$ candidate in a second Galactic GC, M62 (NGC 6266; D = 6.8 kpc; Harris 1996). We call this source M62-VLA1. Unlike the case for the M22 sources, M62-VLA1 has clear X-ray and optical counterparts, and so is the most compelling candidate black hole X-ray binary in a Milky Way GC.
In Section 2, we describe our VLA observations, along with archival Chandra and HST imaging. In Section 3, we present evidence that M62-VLA1 is an accreting stellar-mass $\mathrm{BH}$. Section 4 discusses the host binary system: the binary separation and binary companion. We assess alternative explanations for M62-VLA1 in Section 5. We summarize our findings in Section 6.

\section{OBSERVATIONS}

\subsection{VLA Radio Data}

We observed M62 with the Karl G. Jansky Very Large Array (VLA) over the time period 2012 Sept $10-16$ as part of the program 12B-073 (P.I. Strader). Ten hours were spent observing the cluster, split among seven blocks of $1-1.75 \mathrm{hr}$ duration, yielding a total of $7 \mathrm{hr}$ on source. We observed in $C$ band with $2 \mathrm{GHz}$ total bandwidth and four polarization products. Of the two basebands of $1 \mathrm{GHz}$ width, one was centered at $5.0 \mathrm{GHz}$ and the other at $7.4 \mathrm{GHz}$. The array was in BnA configuration, giving a resolution of $1^{\prime \prime} .4 \times 1^{\prime \prime} .1$ at $5.0 \mathrm{GHz}$. The field of view (full-width at half power) of the VLA at the average frequency of $6.2 \mathrm{GHz}$ was $\sim 7.3$ in diameter, significantly larger than the half-light diameter of M62 (1'.8; Harris 1996).

We observed J1700-2610 as the secondary phase calibrator and $\mathrm{J} 1407+2827$ as the polarization leakage calibrator. 3C286 was used as an absolute flux density, bandpass, and polarization angle calibrator. The data were reduced using standard routines in AIPS. Weights from switched power measurements were applied using TYAPL. Each observing block was edited for bad data and interference and then calibrated. For each individual calibrated baseband, the data were concatenated in the $u v$ plane and then imaged with a Briggs robust value of 1 . A bright source at the edge of the field called for phase and amplitude self calibration. Figure 1 shows a deep co-added image of both basebands, obtained by smoothing the $7.4 \mathrm{GHz}$ baseband to the resolution of the $5.0 \mathrm{GHz}$ basebands and averaging these together. The rms sensitivity of this co-added image is $2.0 \mu \mathrm{Jy}^{\text {beam }^{-1}}$.

M62-VLA1 is the only significant radio source in the core of M62. We use the HST-based core radius from Noyola \& Gebhardt (2006) of $r_{c}=6.16$, and the updated center in J2000 coordinates of $17^{\mathrm{h}} 01^{\mathrm{m}} 13^{\mathrm{s}} .0,-30^{\circ} 06^{\prime} 48^{\prime \prime} .2$ from Lützgendorf et al. (2013). M62-VLA1 is located at $17^{\mathrm{h}} 01^{\mathrm{m}} 13.217,-30^{\circ} 06^{\prime} 50^{\prime \prime} \cdot 60$, 
Table 1

Model Fits M62-VLA1 in Chandra Data

\begin{tabular}{|c|c|c|c|c|c|c|}
\hline Model & $\begin{array}{c}N_{\mathrm{H}}^{\mathrm{a}} \\
\left(10^{21} \mathrm{~cm}^{-2}\right)\end{array}$ & $\Gamma$ & $\begin{array}{c}L_{X}^{\mathrm{b}} \\
\left(\operatorname{erg~s}^{-1}\right)\end{array}$ & $\begin{array}{c}k T \\
(\mathrm{keV})\end{array}$ & $\begin{array}{l}L_{X, \text { therm }} \\
\left(\mathrm{erg} \mathrm{s}^{-1}\right)\end{array}$ & $\chi^{2} / v$ \\
\hline power-law & 2.8 & $2.5 \pm 0.1$ & $3.3 \times 10^{32}$ & . & & $37.7 / 29$ \\
\hline power-law + NS atmosphere & 2.8 & $1.8 \pm 0.3$ & $5.9 \times 10^{32}$ & $0.08 \pm 0.01$ & $2.1 \times 10^{32}$ & $27.5 / 27$ \\
\hline
\end{tabular}

Notes.

${ }^{a}$ Held fixed at the estimated foreground value.

${ }^{\mathrm{b}}$ In the energy range $0.5-7 \mathrm{keV}$.

with positional uncertainty of $0{ }^{\prime} .05$ in both coordinates. It is offset from the cluster center by $3^{\prime \prime} .7(0.12 \mathrm{pc}$, assuming a distance to M62 of $6.8 \mathrm{kpc}$; Harris 1996). There is an additional radio source somewhat outside M62's core, at a cluster radius of 19'. 1. It is a known binary millisecond pulsar, PSR J1701-3006A (Possenti et al. 2003).

Flux density measurements were carried out separately on each baseband's image. We fit Gaussians using JMFIT in AIPS assuming the source is point-like. Measured flux densities for M62-VLA 1 are $19.9 \pm 3.2 \mu \mathrm{Jy}(5.0 \mathrm{GHz})$ and $18.1 \pm 2.3 \mu \mathrm{Jy}$ $(7.4 \mathrm{GHz})$. Assuming that the flux densities follow a power law of the form $S_{v}=v^{\alpha}$, then the spectral index of M62-VLA1 is $\alpha=-0.24 \pm 0.42$. In contrast, PSR J1701-3006A was measured to have a $5.0 \mathrm{GHz}$ flux density of $23.6 \pm 2.9 \mu \mathrm{Jy}$, but was not detected at $7.4 \mathrm{GHz}(3.7 \pm 2.3 \mu \mathrm{Jy})$, implying $\alpha \lesssim-2.0$. The spectrum of M62-VLA1 is inconsistent with the steep spectrum expected for a pulsar.

\subsection{Chandra X-Ray Data}

The archival Chandra imaging was obtained in 2002 May with ACIS-S and a total integration time of $63 \mathrm{ks}$ (Obs ID 2677, P.I. Lewin). M62 hosts a rich population of X-ray sources, with 51 sources detected within the half-mass cluster radius in the Chandra image and only 2-3 expected to belong to the background (Pooley et al. 2003). However, the properties of the individual sources have not been published.

M62-VLA1 is the only X-ray source in M62 which has a counterpart in our VLA image (Figure 1). It is detected in the Chandra image at high significance, with 688 counts or a signalto-noise ratio of 26.

We fit the X-ray spectrum of M62-VLA in the energy range $0.5-7 \mathrm{keV}$ (experiments with other energy ranges yielded very similar results). The foreground absorption was held constant at $N_{\mathrm{H}}=2.8 \times 10^{21} \mathrm{~cm}^{-2}$, calculated from the reddening estimate for M62 $(E(B-V)=0.47$; Harris 1996) and using the Güver \& Özel (2009) relation between reddening and $N_{\mathrm{H}}$. The spectrum was binned so that there were at least 20 counts per channel, and then fit via $\chi^{2}$ minimization in XSPEC.

Assuming a power-law form to the spectrum (as expected for an accreting $\mathrm{BH}$ in the low/hard state), we found a photon index $\Gamma=2.5 \pm 0.1$ (90\% confidence interval) and a $0.5-7 \mathrm{keV}$ luminosity of $\sim 3 \times 10^{32} \mathrm{erg} \mathrm{s}^{-1}$ (Table 1). The fit has a $13 \%$ null hypothesis probability $\left(\chi^{2} / v=1.30\right.$ with 29 degrees of freedom).

The spectrum was not well-fit by an absorbed blackbody $\left(\chi^{2} / v=5.17\right.$ with 29 degrees of freedom). However, we did find a reasonable fit with a combined blackbody and powerlaw spectrum, as observed for accreting neutron stars. The parameters for this model are listed in Table 1; the power law component accounts for about $\sim 2 / 3$ of the flux and the best- fit blackbody temperature is $1.9 \times 10^{6} \mathrm{~K}$. A similarly good fit can be achieved by fitting a model neutron star atmosphere plus a power law, using a NSATMOS model in XSPEC (Heinke et al. 2006) and assuming a neutron star mass of $1.4 M_{\odot}$ and radius of $12 \mathrm{~km}$. The best-fit parameters are again listed in Table 1; the luminosity is practically identical to the power-law + blackbody model, but the temperature of the best-fit neutron star atmosphere is lower.

These fits emphasize the well-known conclusion that it is challenging to distinguish BHs from neutron stars on the basis of X-ray spectra alone.

\subsection{HST Optical Data}

There are archival Hubble Space Telescope (HST)/Advanced Camera for Surveys (ACS) data that cover the core of M62, obtained in 2004 (two years after the Chandra data discussed in Section 2.2, but more than eight years before the VLA data were observed). These data comprised $880 \mathrm{~s}$ of exposure time in F435W ( $B$-equivalent; split into 3 frames), $1170 \mathrm{~s}$ in $\mathrm{F} 625 \mathrm{~W}$ ( $R$-equivalent; 5 frames), and $3610 \mathrm{~s}$ in F658N (narrow band covering $\mathrm{H} \alpha$; 10 frames).

We photometered the individual frames using DOLPHOT (Dolphin 2000) before producing a matched photometric catalog. We note that M62 has a large and differential foreground reddening with mean $E(B-V)=0.47$ (Alonso-García et al. 2011); all photometry quoted in this paper is uncorrected for reddening.

To match the astrometry of the VLA images, we iteratively corrected the astrometry of the HST images to the International Celestial Reference System using a large number of Two Micron All Sky Survey sources across the images. The overall uncertainty in the astrometric zero point of the image is about 0.15 . The uncertainty in the position of M62-VLA1 itself is 0.07 , giving a combined $1 \sigma$ uncertainty of 0.17 in matching the HST and VLA data. Unfortunately, there are no additional sources that appear in both the HST and VLA or Chandra images, so further checks on the astrometry are not possible.

Figure 2 shows the position of M62-VLA1 superposed on the HST F435W image. Even given the significant astrometric uncertainty, M62-VLA1 is a very close $\left(<00^{\prime} .05\right)$ match to a moderately bright star in the images. This object has mean $\mathrm{F} 625 \mathrm{~W}=17.38 \mathrm{mag}$, and $\mathrm{F} 435 \mathrm{~W}-\mathrm{F} 625 \mathrm{~W}=1.41$. In a F625W versus F435W - F625W color-magnitude diagram, the star falls near the lower red giant branch of M62, but lies $\sim 0.4$ mag blueward of the cluster fiducial (Figure 3).

The F625W $-\mathrm{F} 658 \mathrm{~N}$ color of the star is $\sim 0.08$ to $0.1 \mathrm{mag}$ redder than the inferred locus of $\mathrm{F} 435 \mathrm{~W}-\mathrm{F} 625 \mathrm{~W}$ giants, calculated by connecting the principal population of red giant branch stars to that of horizontal branch stars. This photometry is consistent with the presence of $\mathrm{H} \alpha$ emission with an equivalent 


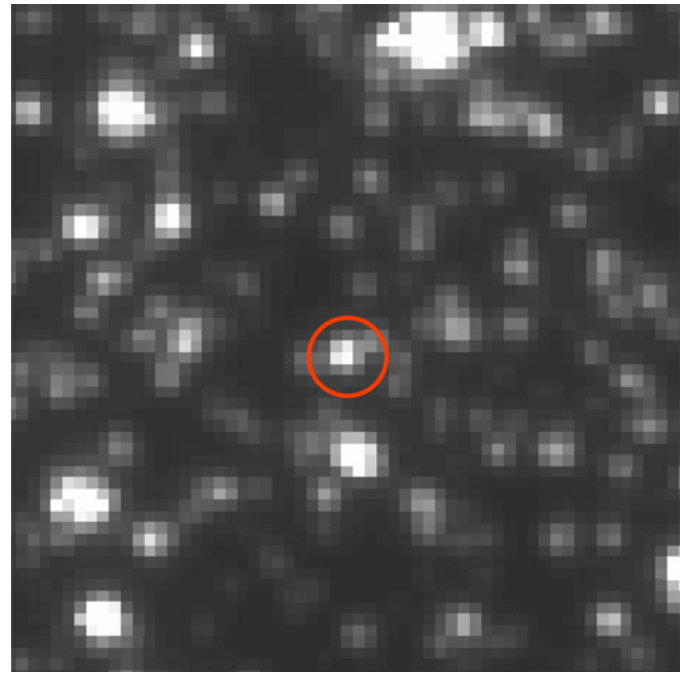

Figure 2. HST F435W image of the candidate optical counterpart to M62-VLA1. The astrometric matching radius ( 0.17$)$ is shown as a red circle. North is up and east is to the left; the image is about $3^{\prime \prime}$ on a side.

(A color version of this figure is available in the online journal.)
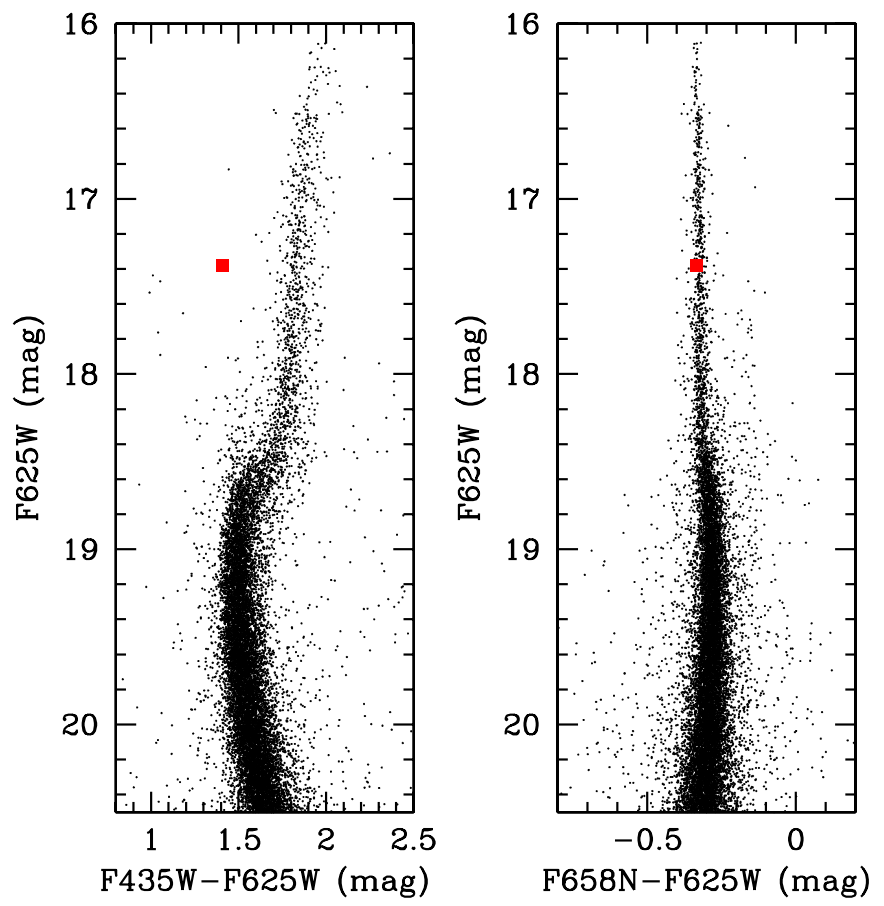

Figure 3. HST color-magnitude diagrams in F625W vs. F435W - F625W (left panel) and F625W vs. F658N - F625W (right panel) of stars in M62. The mean photometry of the M62-VLA1 candidate optical counterpart discussed in Section 2.3 is plotted as a red square. The horizontal branch would be located at $\mathrm{F} 625 \mathrm{~W} \lesssim 16 \mathrm{mag}$, but most of these stars are saturated in the F625W photometry.

(A color version of this figure is available in the online journal.)

width of $\sim 7 \AA$ in the F658N filter. Figures 3 and 4 show color-magnitude and color-color diagrams with the position of this star marked.

This brighter star is flanked by two fainter stars that also fall just within the nominal astrometric error circle (red region in Figure 2). These other stars have photometry consistent with main sequence stars just below the turnoff of M62.

There is some evidence that the brighter candidate optical counterpart star is variable. The 10 frames of $\mathrm{F} 658 \mathrm{~N}$

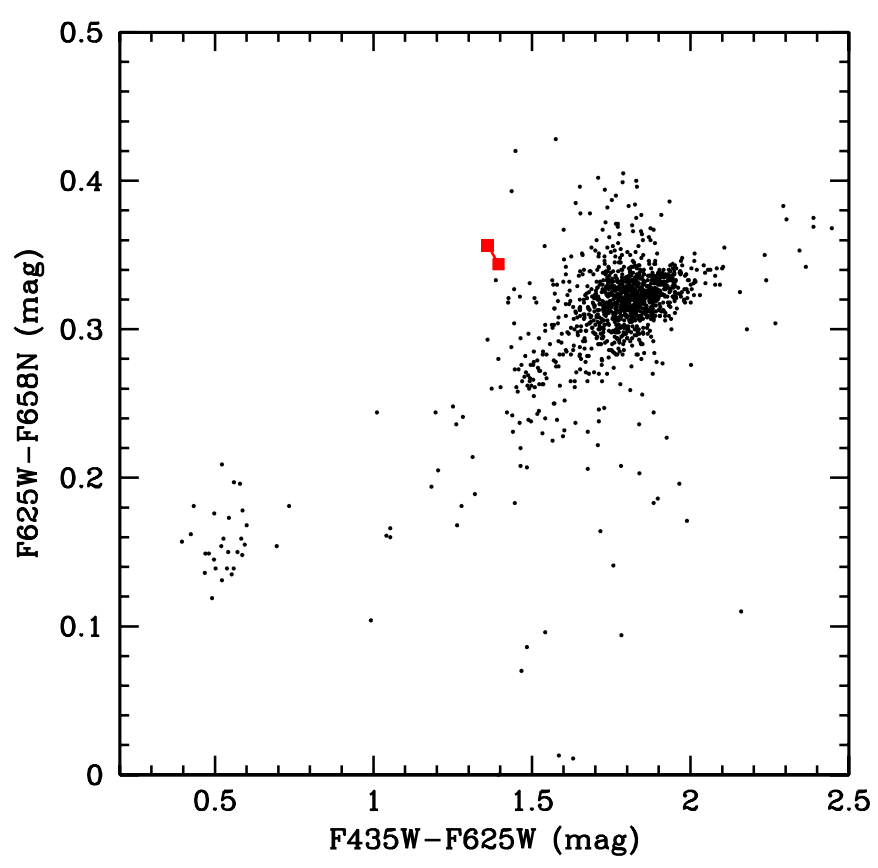

Figure 4. HST F625W - F658N vs. F435W - F625W color-color diagram of unsaturated stars with $\mathrm{F} 625 \mathrm{~W}<19$ mag in the central regions of M62. The candidate optical counterpart of M62-VLA1 (red squares; data at two epochs) is $\sim 0.09$ mag more luminous in F658N than the main locus of stars. One explanation is that this deviation is due to the presence of $\mathrm{H} \alpha$ emission in the F658N band with an equivalent width of $\sim 7 \AA$.

(A color version of this figure is available in the online journal.)

Table 2

Photometry of Candidate Optical Counterpart to M62-VLA1

\begin{tabular}{lll}
\hline \hline $\begin{array}{l}\text { MJD } \\
\text { (days) }\end{array}$ & $\begin{array}{l}\text { Phot. } \\
(\mathrm{mag})\end{array}$ & Filter \\
\hline 53218.25824 & 17.021 & F658N \\
53218.26403 & 17.022 & F658N \\
53218.26983 & 17.004 & F658N \\
53218.31998 & 17.044 & F658N \\
53218.32590 & 17.066 & F658N \\
53218.33181 & 17.047 & F658N \\
53218.38188 & 17.085 & F658N \\
53218.38750 & 17.072 & F658N \\
53218.39313 & 17.079 & F658N \\
53218.39892 & 17.061 & F658N \\
\hline 53218.25199 & 18.773 & F435W \\
53218.31368 & 18.791 & F435W \\
53218.37653 & 18.820 & F435W \\
\hline 53218.24763 & 17.372 & F625W \\
53218.30880 & 17.396 & F625W \\
\hline
\end{tabular}

Notes. All photometry is on the VEGAMAG system and is not corrected for foreground reddening. Uncertainties on the photometry (dominated by systematic effects in the crowded core) are $\sim 0.01 \mathrm{mag}$.

${ }^{a}$ Modified Julian Date at midpoint of exposure.

photometry, acquired over $3 \mathrm{hr}$, show a linear trend to fainter magnitudes, with amplitude $\sim 0.06$ mag over this time period (see Table 2, which lists all photometry of this star, and Figure 5). To assess the significance of this trend, we examined the photometry of stars of comparable brightness $(16.5<\mathrm{F} 658 \mathrm{~N}<17.5 \mathrm{mag})$. For these comparison stars, on average, there is no evidence for a systematic trend in the photometry among the frames. If we fit linear relations to the 


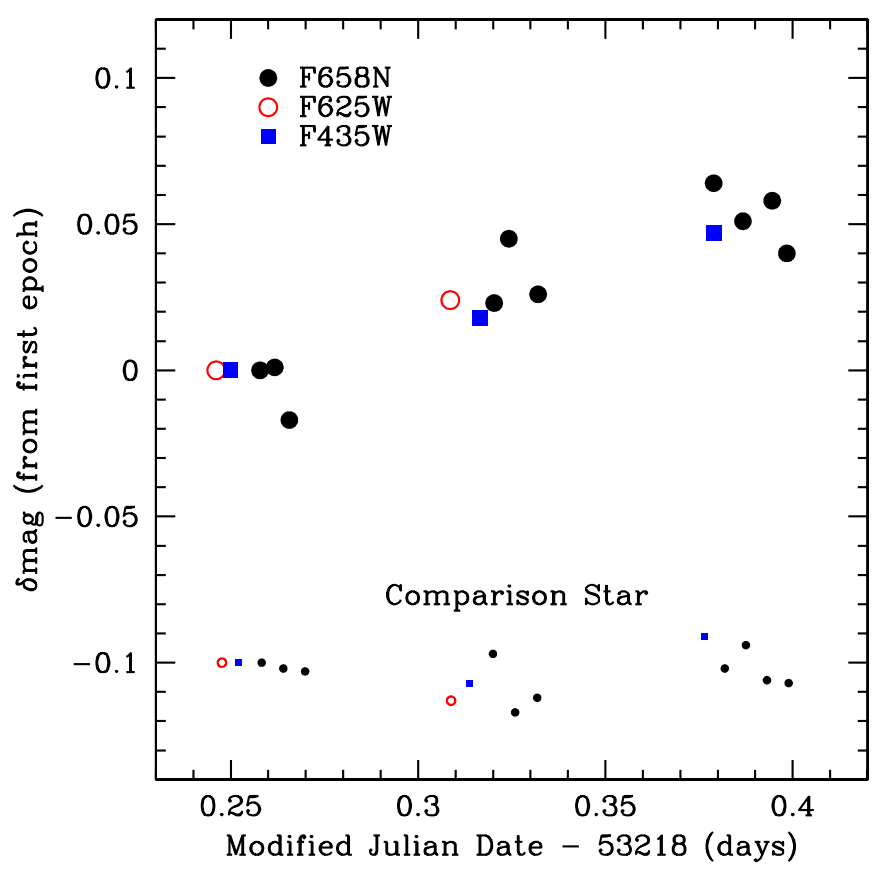

Figure 5. HST optical variability of the likely counterpart to M62-VLA1. The ACS data, taken over 0.15 days $(3.6 \mathrm{hr})$, shows a clear linear trend in three different filters. The star is more variable than $98 \%$ of stars of comparable brightness, lending credence to the interpretation that the source is an accreting binary. Photometry of a randomly chosen comparison star of similar F658N magnitude is shown at bottom with an arbitrary offset.

(A color version of this figure is available in the online journal.)

F658N magnitudes as a function of time for all the comparison stars, the inferred amplitude of the trend for the M62-VLA1 candidate counterpart is larger than $98 \%$ of the stars. The three F435W frames cover a comparable time baseline and show a similar trend, although with a somewhat smaller amplitude. Due to saturation, only two of five frames in F625W have reliable photometry, but these are consistent with the behavior observed in the other filters.

Because of the crowding in the core of M62 and the relatively modest amplitude of the variability, we consider this finding worthy of further investigation but far from conclusive.

\section{A NEW BLACK HOLE CANDIDATE}

The most convincing evidence supporting a BH identification for M62-VLA1 is its ratio of X-ray luminosity $\left(L_{X}\right)$ to radio luminosity $\left(L_{R}\right)$. Figure 6 plots M62-VLA1 on the standard X-ray-radio correlation (Gallo et al. 2006). Its mean flux density of $19 \mu \mathrm{Jy}$ corresponds to an equivalent $8.4 \mathrm{GHz}$ radio luminosity of $L_{R}=9 \times 10^{27} \mathrm{erg} \mathrm{s}^{-1}$ at the distance of M62, assuming a flat spectrum $(\alpha=0$; if the measured $\alpha=-0.24$ is used instead, then $\left.L_{R}=8 \times 10^{27} \mathrm{erg} \mathrm{s}^{-1}\right)$. A spectral fit to the X-ray data gives a luminosity of $L_{X}=7 \times 10^{31} \mathrm{erg} \mathrm{s}^{-1}$ over $3-9 \mathrm{keV}$. This particular radio frequency and $\mathrm{X}$-ray spectral range are chosen because they are the most commonly used in $L_{X}-L_{R}$ relations in the literature.

With the important caveat that variability could be present between the X-ray and radio epochs, M62-VLA1 sits squarely on the $\mathrm{BH} L_{X}-L_{R}$ relation. The source appears to be a doppelganger for the quiescent $\mathrm{BH} \mathrm{V404} \mathrm{Cyg} \mathrm{in} \mathrm{its} \mathrm{X-ray}$ and radio luminosities (Miller-Jones et al. 2009). The radio luminosity for M62-VLA1 is $\gtrsim 2$ orders of magnitude higher than expected for accreting neutron stars or white dwarfs.

Both the radio and X-ray spectra of M62-VLA1 are consistent with an accreting stellar-mass $\mathrm{BH}$. The radio spectral index is consistent with being flat $(\alpha=-0.2 \pm 0.4)$, similar to the radio indices of known low-luminosity accreting stellar-mass BHs ( $\alpha=0.0-0.2$; Fender 2001; Gallo et al. 2005). However,

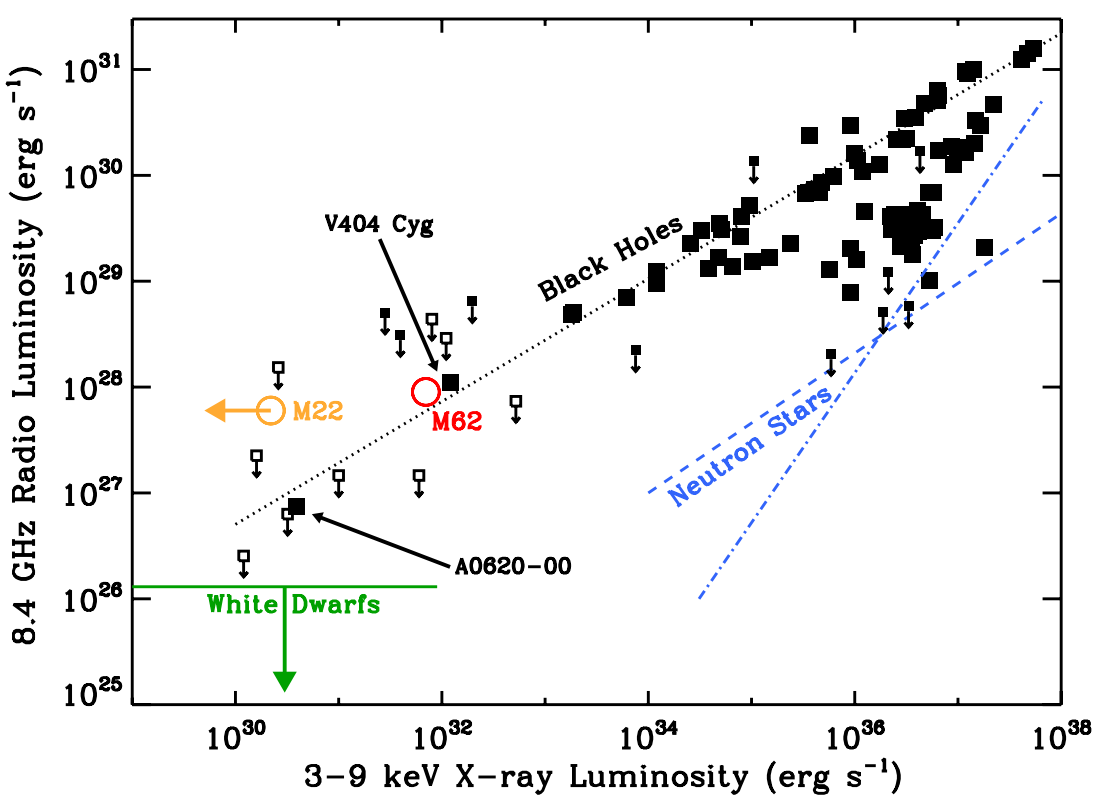

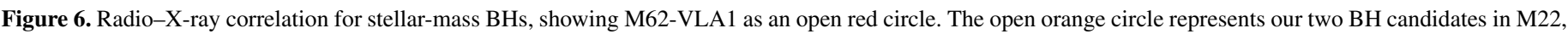

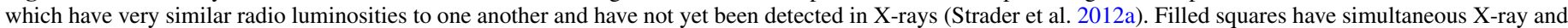

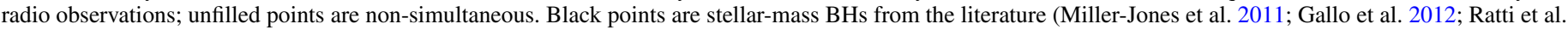

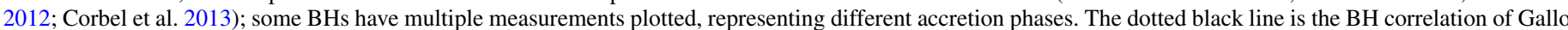

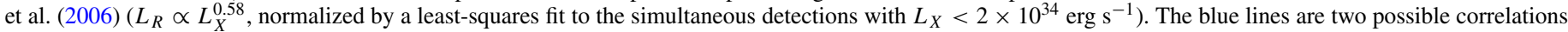

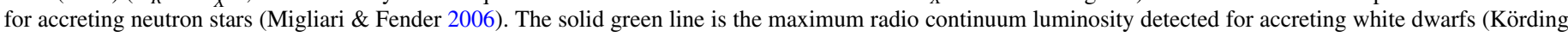
et al. 2008, 2011).

(A color version of this figure is available in the online journal.) 
the radio spectral index could also be consistent with a flaring neutron star, which can have a radio spectrum that varies from $\alpha \approx-0.6$ as expected for optically-thin synchrotron emission to slightly inverted ( $\alpha>0$; e.g., Marti et al. 1992; Fender 1997; Moore et al. 2000; Miller-Jones et al. 2010).

As described in Section 2.2, we also cannot rule out a neutron star origin for M62-VLA1 from the X-ray spectra alone. Either a single power-law or a power-law + thermal model is a good match to the X-ray data. The power-law spectral fit for M62-VLA1 $(\Gamma=2.50 \pm 0.14)$ is comparable to that of the quiescent BH binaries V404 Cyg $(\Gamma=2.17 \pm 0.13$; Corbel et al. 2008), A0620-00 ( $\Gamma=2.1_{-0.4}^{+0.5}$; Gallo et al. 2006), and MAXI J1659-152 ( $\Gamma=2.5 \pm 0.3$; Jonker et al. 2012), although it is slightly softer than commonly observed. The X-ray spectrum of M62-VLA1 is consistent with the claim that the low-luminosity BHs display a softening of their X-ray spectra in quiescence (Corbel et al. 2006; Wu \& Gu 2008).

At a projected distance of 3 .'7 from the photometric center (0.12 pc at the distance of M62), the separation of M62VLA1 is too large to be consistent with an intermediate-mass BH $\left(\sim 10^{2}-10^{4} M_{\odot}\right)$ : such sources would be expected to be $\lesssim 1^{\prime \prime}$ from the center (Chatterjee et al. 2002; van der Marel \& Anderson 2010; Strader et al. 2012b). A caveat is that the photometric center could be offset from the dynamical center, but other evidence disfavors an intermediate-mass $\mathrm{BH}$ interpretation. For example, the fundamental plane of accreting BHs (Merloni et al. 2003) implies that an intermediate-mass BH should have a much higher $L_{R} / L_{X}$ than a stellar-mass $\mathrm{BH}$.

\section{THE HOST BINARY SYSTEM}

Assuming that M62-VLA1 is a stellar-mass BH in M62, the intracluster gas density is very unlikely to be high enough to produce the observed radio and X-ray luminosity via Bondi accretion. Therefore, M62-VLA1 must be accreting from a binary companion. Assuming a nominal $\mathrm{BH}$ mass of $10 \mathrm{M}_{\odot}$, M62-VLA1 would be accreting at $\sim(2-3) \times 10^{-7} L_{\text {edd }}$.

The relatively high quiescent X-ray luminosity of M62VLA1, coupled with the period-luminosity correlation for BH binaries (Garcia et al. 2001; Reynolds \& Miller 2011), suggests a longer orbital period than expected for a dwarf secondary. A giant donor is more likely. This would be yet another similarity between M62-VLA1 and V404 Cyg (which has a long orbital period for a $\mathrm{BH}$ binary at 6.5 days; Casares et al. 1992).

Consistent with this interpretation, the $H S T$ data discussed in Section 2.3 reveals a candidate optical counterpart consistent with a star on the lower red giant branch of M62. However, the F435W - F625W color of the star is unusually blue for an M62 giant, which could indicate the contribution of thermal emission from an accretion disk around the black hole. The photometry also suggests the presence of $\mathrm{H} \alpha$ emission, which could arise from an accretion disk.

In fact, the properties of the possible optical counterpart to M62-VLA1 -including its unusual color-present a reasonable resemblance to V404 Cyg. Casares et al. (1993) estimate that for V404 Cyg, $\sim 26 \%$ and $\sim 9 \%$ of the $B$ - and $R$-band light, respectively, is attributable to accretion. Given these values, which are uncertain by perhaps a factor of two, the star is more than 0.2 mag bluer in $B-R$ than it would be without the presence of an accretion disk (compare this to the $\sim 0.4$ mag offset inferred for M62-VLA1). The equivalent width of $\mathrm{H} \alpha$ emission in V404 Cyg is $\sim 20 \AA$, although somewhat time variable (Hynes et al. 2002, 2009). From our broadband and F658N photometry of M62-VLA1, we estimate a rough $\mathrm{H} \alpha$ equivalent width of $\sim 7 \AA$.

Optical variability often accompanies accreting binaries (see Edmonds et al. 2003 for binaries in 47 Tucanae), and the HST variability observed in all three filters (including the F658N filter covering $\mathrm{H} \alpha$; see Section 2.3 and Figure 5) supports the identification of the candidate giant counterpart with the radio source M62-VLA1.

For comparison, consider V404 Cyg, which exhibits variability on a wide range of time scales, from minutes to days (Casares et al. 1993; Pavlenko et al. 1996; Shahbaz et al. 2003; Zurita et al. 2004). In addition to its 6.5 day orbital period that produces ellipsoidal variations, it shows a $\sim 6 \mathrm{hr}$ quasi-periodic oscillation with variable amplitude, 0.05-0.25 mag (Pavlenko et al. 1996); a similar mode might explain the variability plotted in Figure 5. In addition, when V404 Cyg varies the $\mathrm{H} \alpha$ and optical continuum usually scale together (Hynes et al. 2002); the behavior is also seen in the proposed optical counterpart to M62-VLA1 (Figure 5).

The association between M62-VLA1 and this optical source could be bolstered by further high-resolution photometry, follow-up spectroscopy, and a proper-motion measurement. It should be kept in mind that M62-VLA1 could instead be associated with a star not detected in the HST images, such as an $\mathrm{M}$ dwarf - although this scenario might be disfavored, as the shorter orbital period would lead to a lower mass accretion rate and thus a lower quiescent X-ray luminosity than observed. The system could also, in principle, be a white dwarf-BH binary at an orbital period such that its quiescent luminosity would match the observations; the first extragalactic BH X-ray binary candidate in a GC is likely to be such a system (Maccarone et al. 2007; Zepf et al. 2008).

\section{ALTERNATIVE EXPLANATIONS}

While the properties of M62-VLA1 are well-described by an accreting stellar-mass $\mathrm{BH}$ in M62, here we consider other possible scenarios which might produce this source. Additional discussion for some of these possibilities can be found in the Supplementary Information of Strader et al. (2012a).

\subsection{Accreting Neutron Star or White Dwarf}

As shown in Figure 6, neutron stars or white dwarfs accreting from binary companions are expected to have much lower $L_{R} / L_{X}$ and $L_{R}$ than M62-VLA1 (Fuerst et al. 1986; Migliari \& Fender 2006; Körding et al. 2008, 2011; Byckling et al. 2010). An exception is the class of symbiotic stars, which are white dwarfs accreting from luminous red giants. Radio emission in symbiotic stars is usually thermal, originating from the ionized red giant wind. Therefore, high $L_{R} / L_{X}$ values are observed in these systems $\left(\log L_{R} / L_{X} \approx-2\right.$ to -1 , defined for a radio frequency of $6.2 \mathrm{GHz}$ and X-ray energy range $0.5-8 \mathrm{keV}$; Maccarone et al. 2012). This ratio is significantly higher than observed for M62-VLA1 $\left(\log L_{R} / L_{X}=-4.7\right)$. Symbiotic stars also show inverted radio spectra $(\alpha \approx 0.6$; Seaquist $\&$ Taylor 1990), inconsistent with M62-VLA1. Finally, the giant star which has been tentatively associated with M62-VLA1 is also less optically luminous than typical symbiotic stars. For these reasons, combined with the fact that there are no known symbiotic stars in GCs, we consider it unlikely that M62-VLA1 is a symbiotic star. 


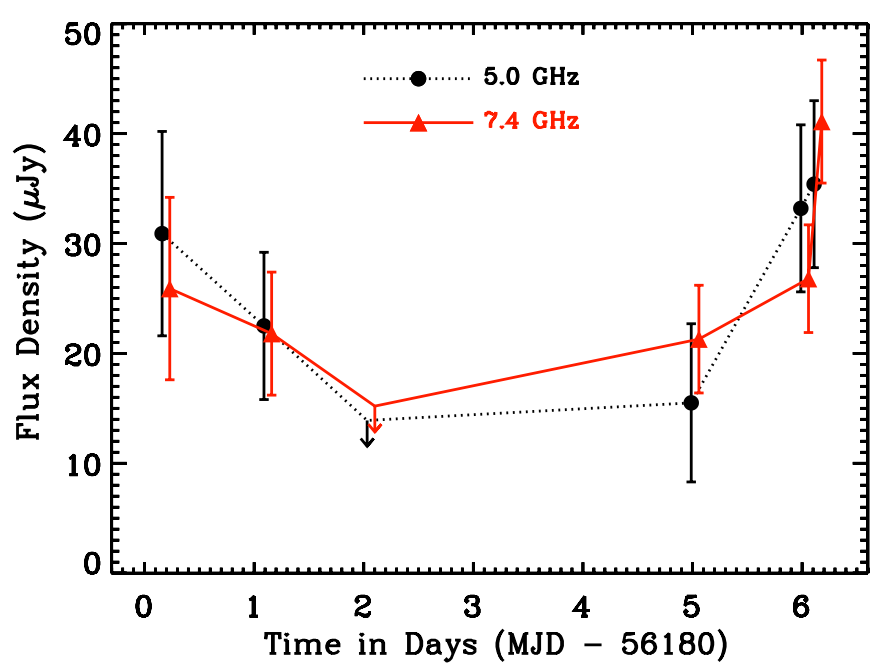

Figure 7. Radio light curve of M62-VLA1 over the week of VLA observations. Black circles connected by a dotted line are $5.0 \mathrm{GHz}$ measurements, while red triangles linked with a dot-dashed line are $7.4 \mathrm{GHz}$ data. Observations are simultaneous at the two frequencies, but the $7.4 \mathrm{GHz}$ data points are offset in time by 0.07 days for clarity. The third epoch, observed on MJD 56182.0, is a non-detection at both frequencies.

(A color version of this figure is available in the online journal.)

We can offer a contrived scenario that could make the observations of M62-VLA1 consistent with that for an accreting neutron star, if we assume the source is strongly variable. The radio and X-ray data on M62 were taken $10 \mathrm{yr}$ apart. The "high" radio luminosity could represent a strong flare on a neutron star, while the accompanying rise in $L_{X}$ may have remained below the detection threshold of X-ray all-sky monitors. The Chandra data would then need to represent a quiescent phase. We note that the duty cycle of neutron star flares is probably low but is poorly constrained.

Strong neutron star outbursts are usually accompanied by a rapid rise and decline in radio luminosity on short timescales (factor of $\gtrsim 3$ in $\sim 4$ days; Moore et al. 2000; Tudose et al. 2009; Miller-Jones et al. 2010). To test this predicted evolution, we imaged each of the VLA observing blocks separately and measured the flux density of M62-VLA1 in six distinct epochs spanning a week (the second and third observing blocks were scheduled directly adjacent to one another and combined into one epoch); the light curve is plotted in Figure 7. M62-VLA1 varies by a factor of $\gtrsim 3$, although its variations are not in the form of a single flare. Instead, the flux density drops over the first two days to a non-detection during the third epoch, and then rises again to reach a maximum in the last epoch. The light curve of M62-VLA1 therefore appears to be inconsistent with a single strong outburst of a neutron star, and is more consistent with the "burbling" observed for quiescent stellar-mass black holes (Miller-Jones et al. 2008). Still, for a secure interpretation of M62-VLA1, simultaneous X-ray and radio data are highly desirable, and are being pursued.

\subsection{Pulsar or Supernova Remnant}

Millisecond pulsars are often found in the cores of GCs, but they display steep radio spectra ( $\alpha \lesssim-1$; Kramer et al. 1999), inconsistent with the measured spectral index of M62-VLA1.

Pulsars in the field occasionally drive a wind that interacts with ambient material, called a pulsar wind nebula. These objects have flat radio spectra, but again are more radio loud than M62-VLA1 $\left(\log L_{R} / L_{X} \approx 0\right.$; Maccarone et al. 2012). In addi- tion, they generally have $L_{X}>10^{34} \mathrm{erg} \mathrm{s}^{-1}$, large diameters, short lifetimes, and dense surroundings, all inconsistent with observations of M62-VLA1 (Gaensler \& Slane 2006). A similar analysis would apply to "redback" systems in which the material originates in an ablated companion (Roberts 2013).

Like pulsar wind nebulae, supernova remnants should be short-lived, angularly extended, and located in dense environments. Supernova remnants in our Milky Way are more radio luminous than M62-VLA1 ( $>0.1 \mathrm{Jy}$ ) and have steeper radio spectra $(\alpha \approx-0.7$; Green 2009 ). For these reasons, we exclude pulsars, pulsar wind nebulae, and supernova remnants as likely identities of M62-VLA1.

\subsection{Planetary Nebula}

Planetary nebulae emit optically thin thermal emission at radio wavelengths, and would therefore show a flat spectral index consistent with M62-VLA1. However, planetary nebulae have low X-ray luminosities $\left(\sim 10^{30} \mathrm{erg} \mathrm{s}^{-1}\right.$; Montez et al. 2010), much fainter than M62-VLA1. In addition, many planetary nebulae are bright in $\mathrm{H} \alpha$, but no nebulosity is observed around M62-VLA in archival HST/WFPC2 images obtained in the F656N filter.

\subsection{Foreground Active Star}

Foreground ( $D \lesssim 100 \mathrm{pc}$ ) coronally active late-type stars can be bright in both the $\mathrm{X}$-ray and radio regimes, show flat radio spectra, and have $\log L_{R} / L_{X} \approx-6$ to -2 (Berger et al. 2010; Maccarone et al. 2012). The candidate optical counterpart of M62-VLA1 is a K giant, not a very late-type dwarf. On the other hand, this match could be spurious and an active dwarf star might escape undetected in the optical imaging if sufficiently distant ( $\gtrsim 50 \mathrm{pc}$ ). To produce detectable radio and X-ray emission at these distances, the star would need to be flaring (Berger et al. 2010). However, a flaring star should also be strongly circularly polarized (Berger 2002; Hallinan et al. 2008), and there is no evidence that M62-VLA1 is circularly polarized ( $3 \sigma$ upper limit $22 \%$ ).

\subsection{Background Galaxy}

It is possible, but very unlikely, that M62-VLA1 is a background radio galaxy. Galaxies exhibit a wide range of $\log$ $L_{R} / L_{X}$ from 0 to -5 (Maccarone et al. 2012). In addition, they show a diversity of radio spectral slopes, although steeper indices than measured for M62-VLA1 are typical $(\alpha \approx-0.7$; Fomalont et al. 2002; Randall et al. 2012). Background galaxies with flat radio spectra are either active galactic nuclei with partially self-absorbed jets, or star-forming galaxies in which the combination of synchrotron and thermal emission produces a flat composite spectrum. Background objects with flux densities as faint as M62-VLA1 ( $20 \mu \mathrm{Jy})$ have not yet been characterized in detail.

We use calculations similar to those in Strader et al. (2012a) to determine the expected number of background sources with properties comparable to those of M62-VLA1. Fomalont et al. (2002) estimate that there are 0.3 sources $\operatorname{arcmin}^{-2}$ with $8.4 \mathrm{GHz}$ flux densities in the range 10-30 $\mu \mathrm{Jy}$. We would therefore expect $\sim 0.01$ sources in the 6." 6 radius core of M62 with similar flux densities as M62-VLA1. This number decreases to $\sim 0.003$ when the spectral index is considered; Fomalont et al. (2002) find that only $30 \%$ of the 47 faint radio sources $(<35 \mu \mathrm{Jy})$ in their sample have $\alpha \geqslant-0.4$, measured from VLA 1.4 and $8.4 \mathrm{GHz}$ data. The lack of a visible background galaxy further reduces 
the probability that M62-VLA1 is a background object: if we conservatively assume that we could detect optical counterparts to $I \approx 21.5$, about a third of the faint flat-spectrum radio sources in the Fomalont et al. sample would have detectable optical matches. On the basis of the radio and optical data, we therefore estimate that the probability that M62-VLA1 is a background galaxy is $<0.003$.

The X-ray counterpart to M62-VLA1 further decreases the probability that it is a background galaxy. For $2-10 \mathrm{keV}$ fluxes equivalent to or brighter than M62-VLA1 $\left(S_{2-10 \mathrm{keV}}>\right.$ $2 \times 10^{-14} \mathrm{erg} \mathrm{s}^{-1} \mathrm{~cm}^{-2}$; Mateos et al. 2008), only $\sim 10^{-3} \mathrm{X}$ ray sources are expected in the core of M62. The X-ray spectrum $(\Gamma=2.5 \pm 0.1)$ is also unusually soft for background sources. Of relatively bright background sources $\left(S_{2-10 \mathrm{keV}} \gtrsim\right.$ $3 \times 10^{-15} \mathrm{erg} \mathrm{s}^{-1} \mathrm{~cm}^{-2}$ ), only $\sim 10 \%$ are fit with $\Gamma \gtrsim 2.2$ (Mateos et al. 2005; Page et al. 2006; Lanzuisi et al. 2013). Therefore, we estimate a very low probability, $\sim 10^{-4}$, that the $\mathrm{X}$-ray counterpart to M62-VLA1 is associated with a background galaxy.

To date we have only investigated four GCs with deep VLA imaging (Strader et al. 2012b, plus M62); two of these GCs have candidate stellar-mass BHs. Therefore, it is unlikely that the detection of a BH look-alike in M62 is due to a "multiple trials" effect. Nonetheless, a direct elimination of the backgroundgalaxy possibility is still needed. As in the accreting neutron star scenario, simultaneous radio and X-ray observations will help distinguish between a stellar-mass $\mathrm{BH}$ and a background galaxy. The fundamental plane of $\mathrm{BH}$ activity suggests that $L_{R} / L_{X} \propto M_{\mathrm{BH}}^{0.38}$ (Merloni et al. 2003). If simultaneous data show a similar ratio as currently observed, then M62-VLA1 is unlikely to be a supermassive $\mathrm{BH}$ in a background active galactic nucleus. In addition, deeper $H S T$ photometry would allow us to search for faint background galaxies coincident with M62-VLA1. Finally, deep high-resolution radio imaging with the High Sensitivity Array would allow us to test if M62-VLA1 shares the proper motion of M62 itself (Dinescu et al. 2003).

\section{CONCLUSIONS AND IMPLICATIONS}

M62-VLA1 is the only radio source in the core of M62 down to flux density levels of $\sim 6 \mu \mathrm{Jy}$ at $5-10 \mathrm{GHz}$. We have argued that it is likely to be a stellar-mass $\mathrm{BH}$ accreting from a binary companion. This scenario is supported by its X-ray and radio luminosities and spectra. The secondary may be a giant star near the base of the red giant branch, as suggested by HST imaging and the $L_{X}$-period relation for known BHs.

While all of our data are consistent with a stellar-mass BH interpretation, other explanations are possible (although unlikely). The most plausible alternative scenarios are (1) an accreting neutron star X-ray binary that is in quiescence during the Chandra observations but flaring during the VLA observations; or (2) a background galaxy. Future observations-principally simultaneous X-ray and radio imaging-will conclusively distinguish between these scenarios.

M62 is now the second Milky Way GC with significant evidence for stellar-mass BHs (see Strader et al. 2012a for discussion of the $\mathrm{BH}$ candidates in M22). We have identified $\mathrm{BH}$ candidates in two out of four GCs with deep VLA radio imaging. While the sample is certainly small, these findings suggest that stellar-mass BHs may be present in many GCs. However, the current sample is not representative of the mass or core density distribution of the Galactic GC system, so our findings are not yet easily generalized. We note that both M62 and M22 are massive GCs, but their structural parameters are quite different. While M22 has a large core radius, M62 has a relatively dense, compact core. This contrast may imply that there are no clear structural diagnostics of the presence BHs in GCs (in contrast with theoretical findings by e.g., Mackey et al. 2008; Sippel \& Hurley 2013; Morscher et al. 2013).

It is worth emphasizing that the BHs which can be detected in accreting binary systems are likely to represent only a small fraction of the population of BHs in a star cluster, since single $\mathrm{BHs}$ and those in binaries not undergoing mass transfer may dominate the BH population in a GC (e.g., Ivanova et al. 2010).

If indeed many GCs host substantial populations of BHs, this represents an important shift in our view of stellar remnants in GCs. The potential implications are large: GCs would become good hunting grounds for new stellar-mass BHs, of which few are still confirmed; BHs in GCs are likely to be more massive than those in the field, opening up a new area of $\mathrm{BH}$ parameter space; it would be possible to determine accurate physical parameters for a significant sample of BHs, since the distances to GCs are accurately known; additional quiescent $\mathrm{BH}$ systems would offer new opportunities to investigate the physics of accretion at the lowest luminosities; and if GCs have multiple $\mathrm{BHs}$, the likelihood of the dynamical formation of $\mathrm{BH}-\mathrm{BH}$ and $\mathrm{BH}$-pulsar systems will increase, improving prospects for the detection of gravitational waves and stringent tests of general relativity. Deep radio imaging of additional GCs, combined with detailed follow-up observations of the candidate BHs in M62 and M22, will determine whether GCs-long thought to be among the least likely hosts for BHs - turn out to be among the best.

We thank S. Sigurdsson and N. Ivanova for useful comments. L. Chomiuk is a Jansky Fellow of the National Radio Astronomy Observatory. J. C. A. Miller-Jones acknowledges support from an Australian Research Council Discovery Grant (DP120102393). C. Heinke is supported by NSERC and an Ingenuity New Faculty Award. The National Radio Astronomy Observatory is a facility of the National Science Foundation operated under cooperative agreement by Associated Universities, Inc. The search for an optical counterpart was based on observations made with the NASA/ESA Hubble Space Telescope, and obtained from the Hubble Legacy Archive, which is a collaboration between the Space Telescope Science Institute (STScI/NASA), the Space Telescope European Coordinating Facility (ST-ECF/ESA) and the Canadian Astronomy Data Centre (CADC/NRC/CSA). The scientific results reported in this article are based to a significant degree on data obtained from the Chandra Data Archive. This work was supported by the NSF through grant AST-1308124.

Facilities: VLA, CXO, HST

\section{REFERENCES}

Aarseth, S. J. 2012, MNRAS, 422, 841

Alonso-García, J., Mateo, M., Sen, B., Banerjee, M., \& von Braun, K. 2011, AJ, 141,146

Altamirano, D., Patruno, A., Heinke, C. O., et al. 2010, ApJL, 712, L58

Altamirano, D., Wijnands, R., Heinke, C. O., Sivakoff, G. R., \& Pooley, D. 2012, ATel, 4264

Berger, E. 2002, ApJ, 572, 503

Berger, E., Basri, G., Fleming, T. A., et al. 2010, ApJ, 709, 332

Blandford, R. D., \& Königl, A. 1979, ApJ, 232, 34

Bozzo, E., Ferrigno, C., Stevens, J., et al. 2011, A\&A, 535, L1

Byckling, K., Mukai, K., Thorstensen, J. R., \& Osborne, J. P. 2010, MNRAS, 408, 2298

Casares, J., Charles, P. A., \& Naylor, T. 1992, Natur, 355, 614 
Casares, J., Charles, P. A., Naylor, T., \& Pavlenko, E. P. 1993, MNRAS, 265,834

Chatterjee, P., Hernquist, L., \& Loeb, A. 2002, ApJ, 572, 371

Corbel, S., Coriat, M., Brocksopp, C., et al. 2013, MNRAS, 428, 2500

Corbel, S., Koerding, E., \& Kaaret, P. 2008, MNRAS, 389, 1697

Corbel, S., Tomsick, J. A., \& Kaaret, P. 2006, ApJ, 636, 971

Dinescu, D. I., Girard, T. M., van Altena, W. F., \& López, C. E. 2003, AJ, 125,1373

Dolphin, A. E. 2000, PASP, 112, 1383

Edmonds, P. D., Gilliland, R. L., Heinke, C. O., \& Grindlay, J. E. 2003, ApJ, 596, 1197

Fender, R. P. 1997, in AIP Conf. Proc. 410, Proceedings of the Fourth Compton Symposium, ed. C. D. Dermer, M. S. Strickman, \& J. D. Kurfess (Melville, NY: AIP), 798

Fender, R. P. 2001, MNRAS, 322, 31

Fomalont, E. B., Kellermann, K. I., Partridge, R. B., Windhorst, R. A., \& Richards, E. A. 2002, AJ, 123, 2402

Fuerst, E., Benz, A., Hirth, W., Kiplinger, A., \& Geffert, M. 1986, A\&A, 154,377

Gaensler, B. M., \& Slane, P. O. 2006, ARA\&A, 44, 17

Gallo, E., Fender, R. P., \& Hynes, R. I. 2005, MNRAS, 356, 1017

Gallo, E., Fender, R. P., Miller-Jones, J. C. A., et al. 2006, MNRAS, 370, 1351

Gallo, E., Miller, B. P., \& Fender, R. 2012, MNRAS, 423, 590

Garcia, M. R., McClintock, J. E., Narayan, R., et al. 2001, ApJL, 553, L47

Green, D. A. 2009, BASI, 37, 45

Güver, T., \& Özel, F. 2009, MNRAS, 400, 2050

Hallinan, G., Antonova, A., Doyle, J. G., et al. 2008, ApJ, 684, 644

Harris, W. E. 1996, AJ, 112, 1487

Heger, A., Fryer, C. L., Woosley, S. E., Langer, N., \& Hartmann, D. H. 2003, ApJ, 591,288

Heinke, C. O., Rybicki, G. B., Narayan, R., \& Grindlay, J. E. 2006, ApJ, 644, 1090

Hynes, R. I., Bradley, C. K., Rupen, M., et al. 2009, MNRAS, 399, 2239

Hynes, R. I., Zurita, C., Haswell, C. A., et al. 2002, MNRAS, 330, 1009

Irwin, J. A., Brink, T. G., Bregman, J. N., \& Roberts, T. P. 2010, ApJL, $712, \mathrm{~L} 1$

Ivanova, N., Chaichenets, S., Fregeau, J., et al. 2010, ApJ, 717, 948

Jonker, P. G., Miller-Jones, J. C. A., Homan, J., et al. 2012, MNRAS, 423, 3308

Kalogera, V., King, A. R., \& Rasio, F. A. 2004, ApJL, 601, L171

Körding, E., Rupen, M., Knigge, C., et al. 2008, Sci, 320, 1318

Körding, E. G., Knigge, C., Tzioumis, T., \& Fender, R. 2011, MNRAS, 418, L129

Kramer, M., Lange, C., Lorimer, D. R., et al. 1999, ApJ, 526, 957

Kulkarni, S. R., Hut, P., \& McMillan, S. 1993, Natur, 364, 421

Kundu, A., Maccarone, T. J., \& Zepf, S. E. 2002, ApJL, 574, L5

Lanzuisi, G., Civano, F., Elvis, M., et al. 2013, MNRAS, 431, 978

Larson, R. B. 1984, MNRAS, 210, 763

Lewin, W. H. G., \& Joss, P. C. 1981, SSRv, 28, 3

Lützgendorf, N., Kissler-Patig, M., Gebhardt, K., et al. 2013, A\&A, 552, A49

Maccarone, T., \& Knigge, C. 2007, A\&G, 48, 5.12

Maccarone, T. J., Kundu, A., Zepf, S. E., \& Rhode, K. L. 2007, Natur, 445,183
Maccarone, T. J., Torres, M. A. P., Britt, C. T., et al. 2012, MNRAS, 426, 3057 Mackey, A. D., Wilkinson, M. I., Davies, M. B., \& Gilmore, G. F. 2008, MNRAS, 386, 65

Marti, J., Paredes, J. M., \& Estalella, R. 1992, A\&A, 258, 309

Mateos, S., Barcons, X., Carrera, F. J., et al. 2005, A\&A, 444, 79

Mateos, S., Warwick, R. S., Carrera, F. J., et al. 2008, A\&A, 492, 51

Merloni, A., Heinz, S., \& di Matteo, T. 2003, MNRAS, 345, 1057

Migliari, S., \& Fender, R. P. 2006, MNRAS, 366, 79

Miller-Jones, J. C. A., Gallo, E., Rupen, M. P., et al. 2008, MNRAS, 388, 1751

Miller-Jones, J. C. A., Jonker, P. G., Maccarone, T. J., Nelemans, G., \& Calvelo, D. E. 2011, ApJL, 739, L18

Miller-Jones, J. C. A., Jonker, P. G., Nelemans, G., et al. 2009, MNRAS, 394, 1440

Miller-Jones, J. C. A., Sivakoff, G. R., Altamirano, D., et al. 2010, ApJL, 716, L109

Montez, R., Jr., De Marco, O., Kastner, J. H., \& Chu, Y.-H. 2010, ApJ, 721,1820

Moody, K., \& Sigurdsson, S. 2009, ApJ, 690, 1370

Moore, C. B., Rutledge, R. E., Fox, D. W., et al. 2000, ApJ, 532, 1181

Morscher, M., Umbreit, S., Farr, W. M., \& Rasio, F. A. 2013, ApJL, 763, L15

Noyola, E., \& Gebhardt, K. 2006, AJ, 132, 447

Page, M. J., Loaring, N. S., Dwelly, T., et al. 2006, MNRAS, 369, 156

Pavlenko, E. P., Martin, A. C., Casares, J., Charles, P. A., \& Ketsaris, N. A. 1996, MNRAS, 281, 1094

Peacock, M. B., Zepf, S. E., Kundu, A., et al. 2012, ApJ, 759, 126

Pooley, D., Lewin, W. H. G., Anderson, S. F., et al. 2003, ApJL, 591, L131

Portegies Zwart, S. F., \& McMillan, S. L. W. 2000, ApJL, 528, L17

Possenti, A., D’Amico, N., Manchester, R. N., et al. 2003, ApJ, 599, 475

Randall, K. E., Hopkins, A. M., Norris, R. P., et al. 2012, MNRAS, 421, 1644

Ratti, E. M., Jonker, P. G., Miller-Jones, J. C. A., et al. 2012, MNRAS, 423, 2656

Reynolds, M. T., \& Miller, J. M. 2011, ApJL, 734, L17

Roberts, M. S. E. 2013, in IAU Symp. 291, Neutron Stars and Pulsars: Challenges and Opportunities after 80 Years, ed. J. van Leeuwen (Cambridge: Cambridge Univ. Press), 127

Seaquist, E. R., \& Taylor, A. R. 1990, ApJ, 349, 313

Shahbaz, T., Dhillon, V. S., Marsh, T. R., et al. 2003, MNRAS, 346, 1116

Sigurdsson, S., \& Hernquist, L. 1993, Natur, 364, 423

Sippel, A. C., \& Hurley, J. R. 2013, MNRAS, 430, L30

Strader, J., Chomiuk, L., Maccarone, T. J., Miller-Jones, J. C. A., \& Seth, A. C. 2012a, Natur, 490, 71

Strader, J., Chomiuk, L., Maccarone, T. J., et al. 2012b, ApJL, 750, L27

Tudose, V., Fender, R. P., Linares, M., Maitra, D., \& van der Klis, M. 2009, MNRAS, 400, 2111

van der Marel, R. P., \& Anderson, J. 2010, ApJ, 710, 1063

van Zyl, L., Charles, P. A., Arribas, S., et al. 2004, MNRAS, 350, 649

Verbunt, F., \& Lewin, W. H. G. 2006, in Globular Cluster X-Ray Sources, ed. W. H. G. Lewin \& M. van der Klis (Cambridge: Cambridge Univ. Press), 341

Wu, Q., \& Gu, M. 2008, ApJ, 682, 212

Zepf, S. E., Stern, D., Maccarone, T. J., et al. 2008, ApJL, 683, L139

Zurita, C., Casares, J., Hynes, R. I., et al. 2004, MNRAS, 352, 877 